\title{
Variabilidade Fisiológica em Populações de Meloidogyne paranaensis
}

\author{
Alexandre D. Roese ${ }^{1}$, Rosângela D.L. Oliveira² ${ }^{2}$ Dagoberto S. Oliveira² \\ 1Embrapa Trigo, CEP 99001-970, Passo Fundo, RS, e-mail: alex@enpt.embrapa.br; ${ }^{2}$ Departamento de Fitopatologia, \\ Universidade Federal de Viçosa, CEP 36.570-000, Viçosa, MG, e-mail: rdlima@ufv.br
}

Autor para correspondência: Alexandre D. Roese

ROESE, A.D., OLIVEIRA, R.D.L. \& OLIVEIRA, D.S. Variabilidade fisiológica em populações de Meloidogyne paranaensis. Fitopatologia Brasileira 32:040-043. 2007.

\begin{abstract}
RESUMO
Comparou-se a capacidade reprodutiva de duas populações de Meloidogyne paranaensis, originárias de plantas de soja (Mp-s) e de cafeeiro (Mp-c), em diferentes hospedeiros. A população Mp-s apresentou maior capacidade reprodutiva que a Mp-c, apresentando fator de reprodução superior em tomateiro e em duas cultivares de soja, porém em cafeeiro a Mpc reproduziu melhor. Em tomateiro 'Santa Clara', ambas reproduziram significativamente mais que nos outros hospedeiros e não houve diferença entre as cultivares de soja 'MS/BR 34' e 'Fepagro RS 10'. Contudo, maior número de populações deverá ser estudado.
\end{abstract}

Palavras-chave adicionais: Glycine max, hospedeiros, virulência de populações, nematóide das galhas.

\begin{abstract}
Physiological variability of two populations of Meloidogyne paranaensis

Two populations of Meloidogyne paranaensis, one from soybean plants (Mp-s) and another from coffee plants (Mp-c) were studied to compare their ability in reproducing on different hosts. Mp-s was able to reproduce more than Mp-c on tomato plants and on two soybean cultivars, but Mp-c showed a higher reproduction factor on coffee plants. On tomato 'Santa Clara', both populations reproduced significantly more than on other hosts, but no difference was detected between the soybean cultivars 'MS/BR 34' and 'Fepagro RS 10'. However, a larger number of populations should be studied.
\end{abstract}

Additional keywords: Glycine max, host, virulence of population, root-knot nematode.

\section{INTRODUÇÃO}

O nematóide formador de galhas Meloidogyne paranaensis Carneiroetal., que sereproduzporpartenogênese mitótica, foi inicialmente detectado em plantas de café no Estado do Paraná. Esta espécie era previamente identificada como M. incognita (Kofoid \& White) Chitwood, e por cerca de duas décadas foi considerada apenas uma variante de $M$. incognita expressando alta virulência ao cafeeiro (Carneiro et al., 1996). Desde a sua descrição como espécie nova, vem apresentando capacidade de parasitar vários hospedeiros, a exemplo do fumo, melancia e tomate (Carneiro et al., 1996), soja (Castro et al., 2003; Roese et al., 2004) e diversas espécies de plantas daninhas, das mais variadas famílias botânicas (Roese \& Oliveira, 2004). Além disto, em ensaios prévios observou-se a existência de variabilidade entre duas populações de $M$. paranaensis quanto à capacidade de formar galhas e produzir ovos em cultivares de soja (Glycine $\max$ L.).

Altos níveis de variação intraespecífica são comuns dentro do gênero Meloidogyne. Essa variabilidade pode ser expressa na morfologia, citogenética, preferência e capacidade reprodutiva em determinados hospedeiros (Roberts \& Thomason, 1989).

Com relação à variação na reprodutividade em hospedeiros selecionados, Zhou et al. (2000) testaram 115 isolados de M. incognita, provenientes de 14 municípios, em três cultivares de algodoeiro resistentes e uma cultivar suscetível a esse nematóide. Observaram que houve efeito de isolados e que dois isolados provenientes de uma das localidades foram mais virulentos aos genótipos de algodoeiro resistentes do que os isolados das demais localidades. Swanson \& Van Gundy (1984), após inocularem separadamente duas cultivares de soja com quatro raças fisiológicas de $M$. incognita, observaram que a interação entre raça e cultivar foi altamente significativa, indicando que a especialização fisiológica de $M$. incognita poderia ser expressa em soja. Roberts et al. (1995) reportaram significativa variação na reprodução de alguns isolados de M. incognita em Vigna sinensis (L.) Endl. que continham o gene de resistência $R k$.

Mesmo M. javanica (Treub) Chitwood, cuja variabilidade tem se mostrado mais restrita do que em $M$. incognita, exibiu diferenças na capacidade de infectar o pimentão (Khan et al., 2003) e amendoim (Centitas et al., 2003). Tzortzakakis (1997) observou variabilidade entre duas populações de $M$. javanica quanto à capacidade de produzirem massas de ovos em duas cultivares de tomateiro resistentes a este nematóide.

Diferenças na capacidade reprodutiva também foram observadas nas espécies Meloidogyne arenaria (Neal) Chitwood (Noe, 1992, Anwar et al., 2000) e M. hapla Chitwood (Goplen et al., 1959; Ogbuji \& Jensen, 1972; Eisenback, 1987; Wofford et al., 1989). 
Ainda que Carneiro \& Jorge (2001) tenham observado alguma seletividade de $M$. paranaensis quando mantida durante sucessivas gerações em plantas de tomateiro ou de cafeeiro, até o momento, não são conhecidas raças fisiológicas nesta espécie. Assim, o objetivo deste trabalho foi comparar a capacidade reprodutiva de duas populações de M. paranaensis, provenientes de regiões e de hospedeiros diferentes, quando cultivares de soja, café e tomate foram inoculadas.

\section{MATERIAL E MÉTODOS}

Duas populações de M. paranaensis, uma proveniente de plantas de soja do município de Vista Alegre, RS e a outra coletada em plantas de café, no município de Patrocínio, MG, foram empregadas neste ensaio. Após a confirmação da identidade, foram multiplicadas em tomateiro cultivar Santa Clara, por, aproximadamente, dois anos no caso da população originária de soja, e por quatro meses para aquela obtida de cafeeiro. Os trabalhos foram conduzidos em casa-de-vegetação do Departamento de Fitopatologia da Universidade Federal de Viçosa.

Antes de ser preparado o inóculo, a pureza das populações foi confirmada. Então, os ovos foram extraídos das raízes, segundo o método de Hussey \& Barker (1973) modificado por Boneti \& Ferraz (1981). A concentração de ovos foi determinada em câmara de contagem de Peters e a suspensão calibrada para $1000 \mathrm{ovos} / \mathrm{ml}$. A inoculação foi feita quando as plantas apresentavam dois pares de folhas completamente desenvolvidas, aproximadamente uma semana após o transplante das mudas para os vasos. Foram utilizados vasos de plástico com capacidade para 2,5 1 , preenchidos com substrato composto de duas partes de solo e uma de areia, previamente esterilizado com brometo de metila. Em cada vaso, contendo uma planta, foram aplicados $5 \mathrm{ml}$ da suspensão de ovos, com o auxílio de uma pipeta, em quatro orifícios, com cerca de $5 \mathrm{~cm}$ de profundidade, feitos no substrato, ao redor das plantas.

Realizou-se a inoculação cruzada com as duas populações de $M$. paranaensis, em quatro hospedeiros, num delineamento do tipo inteiramente casualizado, em esquema fatorial $4 \times 2$, com seis repetições. Os hospedeiros foram soja 'MS/BR 34' e 'Fepagro RS 10', tomateiro 'Santa Clara' e cafeeiro 'Catuaí Vermelho IAC 44', espécies já reconhecidas como boas hospedeiras de $M$. paranaensis. Após 55 dias da inoculação, as plantas foram retiradas dos vasos e as contagens dos números de galhas e massas de ovos realizadas em todos os sistemas radiculares. Posteriormente, as raízes foram submetidas à extração de ovos e juvenis (J2) para a avaliação do número de unidades, permitindo calcular o fator de reprodução do nematóide ( $\mathrm{FR}$ = população final dividida pela população inicial) conforme Oostenbrink (1966). Os dados foram transformados em $\sqrt{x}$ e as médias dos tratamentos comparadas pelo teste de Tukey a $5 \%$ de probabilidade. A análise estatística foi realizada utilizando-se o programa SAEG (Universidade Federal de Viçosa, 2001).

\section{RESULTADOS E DISCUSSÃO}

Meloidogyne paranaensis apresentou variabilidade quanto à capacidade de se reproduzir nos hospedeiros estudados e a análise estatística mostrou significância para cada um dos fatores, população do nematóide e hospedeiro, e para a interação.

De maneira geral, a população originária da soja foi capaz de induzir mais galhas, produzir mais massas de ovos e ovos do que a originária do cafeeiro (Tabelas 1, 2 e 3 ), independente do hospedeiro. O fator de reprodução da população de $M$. paranaensis oriunda da soja foi superior ao da população proveniente do cafeeiro, exceto quando o hospedeiro foi o cafeeiro (Tabela 3). Apenas no cafeeiro, a população originária desse hospedeiro foi capaz de produzir massas de ovos em número significativamente maior do que a população coletada em plantas de soja (Tabela 2 ).

Independente da origem da população, o tomateiro foi melhor hospedeiro do que ambas as cultivares de soja, as quais não diferiram estatisticamente entre si quanto às variáveis número de galhas, número de massas de ovos e número de ovos, exceto para o número de galhas induzidas pela população oriunda de soja. $\mathrm{O}$ cafeeiro foi o pior hospedeiro para M. paranaensis (Tabelas 1, 2 e 3). A população oriunda de cafeeiro apresentou resultados semelhantes para galhas, massas de ovos e ovos quando se comparou o cafeeiro e as duas cultivares de soja, mas esses valores foram superiores nas plantas de tomateiro. Para as duas populações de $M$. paranaensis estudadas, o fator de reprodução, o número de galhas e o número de massas de ovos e de ovos foram maiores em tomateiro 'Santa Clara' do que nas cultivares de soja e no cafeeiro, exceto para o número de galhas da população oriunda de soja.

Sabe-se que o tomateiro é, de maneira geral, um bom hospedeiro para os nematóides do gênero Meloidogyne, tanto que o Projeto Internacional de Meloidogyne (Sasser, 1979) o recomendava para a multiplicação de inóculo em casa-de-vegetação. Mesmo que $M$. paranaensis tenha se reproduzido mais em tomateiro que em soja, sob condições controladas, deve-se salientar que a população oriunda da soja foi mantida por cerca de dois anos em tomateiro, resultando numa adaptação que poderia permitir obter um fator de reprodução cerca de duas vezes maior àqueles obtidos nas cultivares de soja. Tal observação já fora feita por Carneiro \& Jorge (2001). Estes autores observaram que populações de $M$. incognita raça 2 e $M$. paranaensis, quando multiplicadas por dois anos consecutivos em tomateiro, perderam significativamente a capacidade de infectar o cafeeiro, e quando essas populações foram multiplicadas em plantas de cafeeiro também reduziram a capacidade de infectar plantas de tomateiro, porém a seletividade fisiológica foi maior quando as populações foram multiplicadas em plantas de tomateiro.

No presente estudo, a população de $M$. paranaensis oriunda de soja, mesmo após cerca de dois anos multiplicandose em plantas de tomateiro, foi capaz de infectar e multiplicar- 
TABELA 1 - Número de galhas causadas em quatro hospedeiros por populações de Meloidogyne paranaensis ${ }^{1}$

\begin{tabular}{lccccc}
\hline \hline \multirow{2}{*}{ População } & \multicolumn{5}{c}{ Hospedeiros } \\
\cline { 2 - 6 } & $\begin{array}{c}\text { Café 'Catuaí } \\
\text { Vermelho IAC 44' }\end{array}$ & $\begin{array}{c}\text { Tomate iro } \\
\text { 'Santa Clara' }\end{array}$ & $\begin{array}{c}\text { Soja } \\
\text { 'MS/BR 34' }\end{array}$ & $\begin{array}{c}\text { Soja } \\
\text { 'Fepagro RS 10' }\end{array}$ & $\begin{array}{c}\text { Média da } \\
\text { Populaç ão }\end{array}$ \\
\hline Oriunda de soja & 2,7 a C & $1.184,8$ a AB & $1.764,5$ a A & 976,3 a B & 982,1 a \\
$\begin{array}{l}\text { Oriunda de café } \\
\text { Média do }\end{array}$ & 17,0 a B & $1.141,0$ a A & 9,0 b B & 58,7 b B & 306,4 b \\
hospedeiro & $\mathbf{9 , 9 ~ C ~}$ & $\mathbf{1 . 1 6 2 , 9 ~ A ~}$ & $\mathbf{8 8 6 , 8 ~ B}$ & $\mathbf{5 1 7 , 5 ~ B ~}$ & \\
\hline
\end{tabular}

${ }^{1}$ Média de seis repetições. Os dados dos números de galhas foram transformados em $\sqrt{x}$ Médias seguidas da mesma letra na coluna (em minúsculo) ou na linha (em maiúsculo) não diferem entre si pelo Teste de Tukey $(\mathrm{P} \leq$ $0,05)$.

TABELA 2 - Número de massas de ovos em quatro hospedeiros, após inoculação com duas populações de Meloidogyne paranaensis ${ }^{1}$

\begin{tabular}{lccccc}
\hline \hline \multirow{2}{*}{ População } & \multicolumn{5}{c}{ Hospedeiros } \\
\cline { 2 - 6 } & $\begin{array}{c}\text { Café 'Catuaí } \\
\text { Vermelho IAC 44' }\end{array}$ & $\begin{array}{c}\text { Tomateiro 'Santa } \\
\text { Clara' }\end{array}$ & $\begin{array}{c}\text { Soja } \\
\text { 'MS/BR 34' }\end{array}$ & $\begin{array}{c}\text { Soja } \\
\text { 'Fepagro RS 10' }\end{array}$ & $\begin{array}{c}\text { Média d a } \\
\text { População }\end{array}$ \\
\hline Oriunda de soja & 0,3 b C & 593,8 a A & 285,2 a B & 402,2 a B & 320,4 a \\
Oriunda de café & 12,0 a B & 416,5 b A & 5,8 b B & 8,3 b B & 110,7 b \\
$\begin{array}{l}\text { Média do } \\
\text { hospedeiro }\end{array}$ & $\mathbf{6 , 2} \mathbf{C}$ & $\mathbf{5 0 5 , 2}$ A & $\mathbf{1 4 5 , 5}$ B & $\mathbf{2 0 5 , 3 ~ B ~}$ & \\
\hline
\end{tabular}

${ }^{1}$ Média de seis repetições. Os dados dos números de massas de ovos foram transformados em $\sqrt{x}$ Médias seguidas da mesma letra na coluna (em minúsculo) ou na linha (em maiúsculo) não diferem entre si pelo Teste de Tukey $(\mathrm{P} \leq$ $0,05)$.

TABELA 3 - Número de ovos produzidos por duas populações de Meloidogyne paranaensis e fator de reprodução (FR) em quatro hospedeiros ${ }^{1}$

\begin{tabular}{lccccc}
\hline \hline População & $\begin{array}{c}\text { Café 'Catuaí } \\
\text { Vermelho IAC 44' }\end{array}$ & $\begin{array}{c}\text { Tomate iro } \\
\text { 'Santa Clara' }\end{array}$ & $\begin{array}{c}\text { Sojpedeiros } \\
\text { Soja } \\
\text { 'MS/BR 34' }\end{array}$ & $\begin{array}{c}\text { Soja } \\
\text { 'Fepagro RS 10' }\end{array}$ & $\begin{array}{c}\text { Média da } \\
\text { População }\end{array}$ \\
\hline Oriunda de soja & $164,2 \mathbf{a C}$ & $749.350,4 \mathbf{a A}$ & $363.972,4 \mathbf{a B}$ & $477.628,4 \mathbf{a B}$ & $397.778,9$ a \\
FR $^{2}$ & 0,0 & 149,9 & 72,8 & 95,5 & \\
Oriunda de café & $5.145,1 \mathbf{a B}$ & $307.231,6 \mathbf{b A}$ & $16.584,8 \mathbf{b B}$ & $18.227,1 \mathbf{b B}$ & $86.797,2 \mathbf{b}$ \\
FR $^{2}$ & 1,0 & 61,4 & 3,3 & 3,6 & \\
$\begin{array}{l}\text { Média do } \\
\text { hospedeiro }\end{array}$ & $\mathbf{2 . 6 5 4 , 7} \mathbf{C}$ & $\mathbf{5 2 8 . 2 9 1 , 0 ~ A}$ & $\mathbf{1 9 0 . 2 7 8 , 6} \mathbf{B}$ & $\mathbf{2 4 7 . 9 2 7 , 8 ~ B}$ & \\
\hline
\end{tabular}

${ }^{1}$ Média de seis repetições. Os dados dos números de ovos foram transformados em $\sqrt{x}$ Médias seguidas da mesma letra na coluna (em minúsculo) ou na linha (em maiúsculo) não diferem entre si pelo Teste de Tukey $(\mathrm{P} \leq 0,05)$.

${ }^{2} \mathrm{FR}=$ população final/população inicial.

se nas duas cultivares de soja testadas, tendo apresentado fatores de reprodução elevados. Este fato pode indicar que esta espécie de nematóide perde mais rapidamente a habilidade de reproduzir em plantas de cafeeiro do que em plantas de soja. Este assunto, porém, necessita ser melhor investigado, comparando-se populações multiplicadas por períodos mais prolongados em tomateiro e em soja.

Embora as diferenças entre as populações provenientes da soja e do cafeeiro sejam significativas para as variáveis estudadas, principalmente massa de ovos e produção de ovos, deve-se considerar que a avaliação do ensaio ocorreu aos 55 dias após a inoculação. Esse tempo, para o cafeeiro, parece não ser suficiente para permitir boa multiplicação dos nematóides das galhas, como já se tem verificado para Meloidogyne exigua Goeldi e M. incognita (Gonçalves et al., 1996; Silvarolla et al., 1998; Silva, 2005). Além disso, no cálculo do fator de reprodução não foram considerados, para a contagem da população final, os juvenis presentes no solo, o que pode ter subestimado esse índice.

Vale ressaltar, no entanto, que a origem do isolado ou população da espécie de nematóide que se está estudando é um fator importante nos estudos de melhoramento, pois genótipos que apresentam resistência e, ou, tolerância a uma determinada espécie, podem apresentar comportamentos diferentes frente a isolados da mesma espécie oriundos de regiões diferentes, como observado por Wofford e colaboradores (1989) e também por Davis e colaboradores (1996). No presente trabalho observouse haver variabilidade fisiológica entre as duas populações de M. paranaensis estudadas, no que se refere à capacidade de reproduzir-se em raízes de diferentes hospedeiros. 


\section{REFERÊNCIAS BIBLIOGRÁFICAS}

ANWAR, S.A., MC KENRI, M.V. \& FADDOUL, J. Reproductive variability of field populations of Meloidogyne spp. on grape rootstocks. Journal of Nematology 32:265-270. 2000.

BONETI, J.I.S. \& FERRAZ, S. Modificação do método de Hussey e Barker para extração de ovos de Meloidogyne exigua de raízes de cafeeiro. Fitopatologia Brasileira 6:553. 1981. (Resumo)

CARNEIRO, R.M.D.G., CARNEIRO, R.G., ABRANTES, I.M.O, SANTOS, M.S.N.A. \& ALMEIDA, M.R.A. Meloidogyne paranaensis n.sp. (Nemata: Meloidogynidae), a root-knot nematode parasitizing coffee in Brazil. Journal of Nematology 28:177-189. 1996.

CARNEIRO, R.M.D.G. \& JORGE, C.L. Seletividade fisiológica de populações de Meloidogyne incognita e Meloidogyne paranaensis quando multiplicadas durante sucessivas gerações em tomateiros e cafeeiros. Anais, $2^{\circ}$ Simpósio de Pesquisa dos Cafés do Brasil. Brasília. DF. 2001. pp. 82-83.

CASTRO, J.M.C., LIMA, R.D. \& CARNEIRO, R.M.D.G. Variabilidade isoenzimática de populações de Meloidogyne spp. provenientes de regiões brasileiras produtoras de soja. Nematologia Brasileira 27:1-12. 2003.

CENTITAS, R., OLIVEIRA, R.D.L., MENDES, M.L., BRITO, J.A. \& DICKSON, D.W. Meloidogyne javanica on peanut in Florida. Journal of Nematology 35:433-436. 2003.

DAVIS, E.L., KOENNING, S.R., BURTON, J.W. \& BARKER, K.R. Greenhouse evaluation of selected soybean germplasm for resistance to North Carolina populations of Heterodera glycines, Rotylenchulus reniformis, and Meloidogyne species. Journal of Nematology 28:590-598. 1996.

EISENBACK, J.D. Reproduction of northern root-knot nematode (Meloidogyne hapla) on marigolds. Plant Disease 71:281. 1987. (Abstract)

GONÇALVES, W., FERRAZ, L.C.C.B., LIMA, M.M.A. \& SILVAROLLA, M.B. Patogenicidade de Meloidogyne exigua e $M$. incognita raça 1 a mudas de cafeeiros. Bragantia 55:89-93. 1996.

GOPLEN, B.P., STANFORD, E.H. \&ALLEN, M.W. Demonstration of physiological races within three root-knot nematode species attacking alfafa. Phytopathology 49:653-656. 1959.

HUSSEY, R.S. \& BARKER, K.R. A comparasion of methods for collecting inocula of Meloidogyne spp., including a new technique. Plant Disease Reporter 57:1025-1028. 1973.

KHAN, B., KHAN, A.A. \& KHAN, M.R. Pathogenic variability among isolates of Meloidogyne javanica on Capsicum annum. Journal of Nematology 35:430-432. 2003.

NOE, J.P. Variability among populations of Meloidogyne arenaria. Journal of Nematology 24:404-414. 1992.
OGBUJI, R.O. \& JENSEN, H.J. Pacific northwest biotypes of Meloidogyne hapla. Plant Disease Reporter 56:520-523. 1972.

OOSTENBRINK, M. Major characteristics of the relation between nematodes and plants. Meded. Landbouwhogeschool. Wageningen 66:3-46. 1966.

ROBERTS, P.A., FRATE, C.A., MATTHEWS, W.C. \& OSTERLI, P.P. Interactions of virulent Meloidogyne incognita and Fusarium wilt on resistant cowpea genotypes. Phytopathology 85:12881295. 1995.

ROBERTS, P.A. \& THOMASON, I.J. A review of variability in four Meloidogyne spp. measured by reproduction on several hosts including Lycopersicon. Agricultural Zoology Review 3:225-252. 1989.

ROESE, A.D. \& OLIVEIRA, R.D.L. Capacidade reprodutiva de Meloidogyne paranaensis em espécies de plantas daninhas. Nematologia Brasileira 28:137-141. 2004.

ROESE, A.D., OLIVEIRA, R.D.L. \& LANES, F.F. Reação de cultivares de soja (Glycine max L. Merril) a Meloidogyne paranaensis. Nematologia Brasileira 28:131-135. 2004.

SASSER, J.N. Pathogenicity, host hanges and variability in Meloidogyne species. In: Lamberti, F. \& Taylor, C.E. (Eds.) Rootknot nematodes (Meloidogyne species) - systematics, biology and control. New York. Academic Press. 1979. pp. 257-268.

SILVA, R.V. Produção de inóculo e diferenciação de raças de Meloidogyne exigua em Coffea spp. Dissertação de Mestrado. Viçosa MG. Universidade Federal de Viçosa. 2005.

SILVAROLLA, M.B., GONÇALVES, W. \& LIMA, M.M.A. Resistência do cafeeiro a nematóides. V - Reprodução de Meloidogyne exigua em cafeeiros derivados da hibridação de Coffea arabica com Coffea canephora. Nematologia Brasileira 22:51-59. 1998.

SWANSON, T.A. \& VAN GUNDY, S.D. Variability in reproduction of four races of Meloidogyne incognita on two cultivars of soybean. Journal of Nematology 16:368-371. 1984.

TZORTZAKAKIS, E.A. Variability in reproduction of Meloidogyne javanica and $M$. incognita on tomato and pepper. Nematropica 27:91-97. 1997.

UNIVERSIDADE FEDERAL DE VIÇOSA. SAEG (Sistema de Análises Estatísticas e Genéticas) - Versão 8.0. Viçosa, MG. Universidade Federal de Viçosa. 2001.

WOFFORD, D.S., GRAY, F.A. \& ECKERT, J.W. Pathogenicity of two populations of Meloidogyne hapla Chitwood on alfafa and sainfoin. Journal of Nematology 21:87-91. 1989.

ZHOU, E., WHEELER, T.A. \& STARR, J.L. Root galling and reproduction of Meloidogyne incognita isolates from Texas on resistant cotton genotypes. Journal of Nematology 32:513-518. 2000. 